\title{
DIFICULTADES \\ INTERGENERACIONALES EN LA COMUNICACIÓN INTERPERSONAL POR EL USO DE LAS TECNOLOGÍAS
}

\section{INTERGENERATIONAL DIFFICULTIES IN INTERPERSONAL COMMUNICATION CAUSED BY USE OF TECHNOLOGY}

\author{
JIMENA LEIVA R. ${ }^{1}$ \\ Recibido: 6 de enero de 2017 \\ Aceptado: 6 de marzo de 2017
}

\footnotetext{
${ }^{1}$ Pontificia Universidad Católica del Ecuador, Facultad de Comunicación, Lingüística y Literatura, Escuela de Comunicación, Quito, Ecuador (jalexleivar@gmail.com).
} 



\section{DIFICULTADES \\ INTERGENERACIONALES EN LA \\ COMUNICACIÓN INTERPERSONAL \\ POR EL USO DE LAS TECNOLOGÍAS}

\section{INTERGENERATIONAL DIFFICULTIES IN INTERPERSONAL COMMUNICATION CAUSED BY USE OF TECHNOLOGY}

JIMENA LEIVA R.

PALABRAS CLAVE: nativos digitales, comunicación interpersonal o cara a cara, inmigrantes digitales cibercultura, adicción.

KEYWORDS: technological natives, interpersonal communication, technological migrants, cyberculture, addiction

\section{RESUMEN}

El presente estudio tuvo como objetivo determinar las dificultades de comunicación interpersonal entre jóvenes (nativos digitales) y personas de otras generaciones (migrantes digitales) por el uso de nuevas formas de comunicación tecnológica, transformando la cotidianidad y prácticas de los jóvenes-. Para ello, fueron encuestados jóvenes universitarios. Los resultados demostraron que estos jóvenes tienen nuevas formas de comunicación que responden a sus intereses y perspectivas. También prefieren conversar por internet con sus pares o personas desconocidas de su misma edad que conversar cara a cara con familiares o personas de otras edades. 


\section{ABSTRACT}

This study has determined the difficulties of interpersonal communication between young people (technological natives) and people of other generations (technological migrants) by using new forms of technological communication. It has led to the transformation of everyday life and practices of young people. This study, which was done to many young university students in Quito, has shown they are part of the technological age. Moreover, it has displayed that they have new communication ways according to their interests and perspectives. They also prefer to talk by internet with their peers or unknown people of the same age instead of talking face-to-face with their relatives or acquaintances of different ages around them.

The new technologies are gradually replacing with direct communication as a daily habit. Furthermore, there are fewer spaces where people could share and dialogue with others. Perhaps, in the future, the great human characteristics will not be displaced by ICT, and persons could keep their capacity of thought and speech.

\section{INTRODUCCION}

Hasta antes de que aparezcan los nuevos medios tecnológicos, los jóvenes, llegaban a la edad adulta con la formación que recibían de su entorno escolar y tradicional, lo que les bastaba para ocupar su lugar en un ambiente familiar y social estático y vivir una vida sin demasiadas preocupaciones. Hoy, los jóvenes se desenvuelven en la sociedad de la información y comunicación, lo que ha modificado su cotidianidad y sus hábitos diarios, esto ha provocado preocupación a nivel mundial por parte de padres de familia, académicos, estudio- sos del tema, porque los nativos digitales crecen con la tecnología y son expertos en navegar por la red, no obstante tienen dificultades para pensar, concentrarse y comunicarse con las otras personas.

Según Sierra (Sierra, 2012) hasta los años 70, muchos jóvenes en el país se juntaban y se vinculaban a actividades religiosas y políticas. Eran parte de organizaciones mucho más grandes como por ejemplo juventudes del partido socialista, no constituían en sí comunidades cerradas, sino por el contrario conformaban grandes comunidades y 
todos los relatos de legitimación los obtenían de ellas, Sierra añade que ahora los jóvenes tienen la conexión con las redes virtuales, el soporte digital que les permite romper con la distancia geográfica y con barreras que antes no era posible porque no había la infraestructura adecuada.

Aquellos jóvenes nacidos en los años 80 en adelante nacen con la era de la computadora y la internet, por lo que su desempeño en el manejo de estas herramientas es eficiente. A estos jóvenes se los conoce como nativos digitales, según la Unicef, ellos comparten una cultura mundial común. (UNICEF, 2011) Tienen facilidades de socialización y comunicación a nivel planetario, no así con los seres que viven cerca de ellos como sus padres o personas adultas, lo que desmejora la comunicación interpersonal.

En la revista Chasqui, en el artículo titulado Jóvenes y tics (CIESPAL, 2012) se destaca que los jóvenes se comunican con sus amigos mediante correos electrónicos y mensajes de telefonía celular, navegan a diario por la red, hacen amigos en los chats y son los que descubren los nuevos usos de las tecnologías, sin embargo en las relaciones interpersonales con personas de otras generaciones tienen dificultades.

Este problema de comunicación es analizado desde el campo de la psicología y la sociología, pero muy poco o casi nada desde la disciplina de la comunicación, de ahí que la mayor parte de literatura que está disponible sobre el tema se ha generado en relación a la adicción que tienen los adolescentes y jóvenes en torno a las nuevas tecnologías. Entendiéndose por adicción como una dependencia una dependencia de sustancias o actividades nocivas para la salud o el equilibrio psíquico. (Real Academia Española , 2014)

Uno de los estudios que se realizó desde el campo de la psicología, en el 2008 en Madrid, determina que la adicción en los jóvenes está causando alarma social, el estudio se llevó a cabo con una muestra de 1710 menores escolarizados de la comunidad de Madrid, (Echeburua, 2009), la edad comprendía entre los 12 a los 17 años, el resultado determinó que la televisión es la más utilizada con el 93,23\% reconocen utilizarla siempre, seguida de la Internet $87,43 \%$ y teléfono móvil $80,81 \%$, desde la variable sexo en la investigación se determina como resultado que las mujeres presentan valores significativamente superiores a los varones en el uso de la Internet y teléfono móvil, pero inferiores en el uso de videojuegos.

El estudio antes mencionado evalúa también los síntomas característicos de las adicciones, y determina en la encuesta que la sintomatología más importante y que aparece con las mayores 
puntuaciones es el alivio que sienten los jóvenes con el uso de las nuevas tecnologías.

Otra investigación realizada en el 2007 (Garitaonandia, 2007) también en España habla de cómo usan la Internet los jóvenes: hábitos, riesgos y control parental", donde se destacan actitudes, hábitos, competencias y comportamientos de los jóvenes, los efectos y las consecuencias de las relaciones de estos con sus iguales o con sus padres.

La realidad antes descrita no es ajena a la sociedad ecuatoriana, especialmente en el área urbana; en los últimos años el país ha tenido que dar un salto significativo en materia de nuevas tecnologías. La evolución de las mismas ha obligado a las personas a incursionar en el mundo digital de distintas maneras, también los niños y jóvenes nacieron con las TICS, (nativos digitales), a diferencia de las personas adultas mayores que siempre usaron el papel o la máquina de escribir, los telegramas para elaborar documentos o comunicarse a largas distancias.

En el caso de nuestro país, hay un estudio que realizó el Laboratorio de medios de la UDLA en febrero del 2009 (Cevallos, 2012), en cinco universidades de Quito, tres privadas y dos públicas, donde se pretendía comprender los cambios culturales en las actitudes y comportamientos de los jóvenes así como conocer las causas que llevaban a los jóvenes a modificar sus conductas en relación, no con el hecho de tener el celular como aparato para comunicarse, sino cómo el aparato móvil que adquiere vida propia y extiende sus funciones a otros espacios.

De acuerdo con datos oficiales proporcionados por el INEC en nuestro país el acceso a Internet se ha incrementado año a año, tal es el caso que hasta el 2015 los jóvenes de 16 a 24 años son los que más usan las tecnologías, con el $67,8 \%$, seguidos de las personas de 5 a 15 años con 58,3\%. (INEC, 2016), además según los mismos datos la provincia con mayor porcentaje de personas que tienen celular activado es Pichincha con el $64,5 \%$ y el $89.90 \%$ es la población que tiene celular y utiliza redes sociales. Estos datos demuestran que la relación de los jóvenes con las nuevas tecnologías es evidente, especialmente aquellos que viven en la urbe.

Los datos que se presentan en este artículo de investigación se obtuvieron en noviembre del 2012, sin embargo es información actual y aporta a la discusión social sobre el uso de las tics en los jóvenes y el manejo de la comunicación cara a cara con personas de otras generaciones. 


\section{OBJETIVO GENERAL:}

Determinar las dificultades de comunicación interpersonal entre jóvenes (nativos digitales) y personas de otras generaciones (migrantes digitales), por el uso de nuevas formas de comunicación tecnológica.

\section{OBJETIVOS ESPECÍFICOS:}

- Examinar las fuentes documentales existentes que aborden la temática objeto del estudio.

- Determinar las dificultades de comunicación "cara a cara", mediante la aplicación de técnicas de investigación que permitan recabar la información en los jóvenes universitarios de la PUCE.

\section{LA COMUNICACIÓN VERBAL Y NO VERBAL}

La comunicación nace con el ser humano, todo significa y de muchas maneras, también comunica. Así la interacción e interrelación de las personas solo son posibles a través de los diversos tipos de comunicación, como por ejemplo lo constituyen los recursos simbólicos tan antiguos y acabados como el lenguaje verbal y no verbal, o aquellos tecnológicos como los medios de comunicación análogos o digitales (Pereira, 2002).

A la comunicación hay que asumirla como una praxis colectiva que se manifiesta a través de formas simbólicas tales como el lenguaje verbal, no verbal, el arte, mitos, etc., además a través de sistemas de significación como la concepción del tiempo y del espacio, la gestualidad, la vestimenta, las ceremonias, los objetos o los colores, cuya importancia radica en la producción, intercambio y percepción de realidades cotidianas, estéticas, científicas, mitológicas y otras (Pereira, 2002).

Según lo señala Alberto Pereira, los hechos culturales se dan cuando las personas interactúan socialmente mediante el trabajo, las prácticas, los hábitos, los lenguajes, etc. y adquieren la capacidad de codificar y simbolizar la realidad mediante diferentes formas y sistemas de interrelación, cuya representación más alta y compleja lo constituye el lenguaje verbal, que es una comunicación no instintiva, aprendida y cultural según lo menciona Sapir, que junto a otros lenguajes y formas no verbales de interacción nos elevan a la categoría de entes socio-culturales(Pereira, 2012:29). 
La comunicación "cara a cara" o interpersonal es la más antigua, a inicios de la humanidad este tipo de comunicación consistía en gritos y gestos, luego creció hasta complejizarse con el lenguaje verbal, que ha sido un factor fundamental para la creación y permanencia de la cultura.

Esa trasmisión oral de generación a generación ha dejado un legado de valores, hábitos y normas, que las personas las ejercen cotidianamente. Del 100\% de la comunicación cara a cara el 65\% es lenguaje no verbal y el 35\% lenguaje oral, lo que significa que el lenguaje no verbal es básico para percibirle al interlocutor desde sus sentimientos, sensaciones, porque intervienen los gestos, la mirada, la expresión corporal y la espontaneidad del sujeto para expresarse.

Además este tipo de comunicación interpersonal tiene como característica específica a lo situacional, es decir que se da en un espacio determinado ubicando el medio social en el que se intercambian los mensajes. Este manejo interpersonal está asociado con la relación, lo que significa según Fernández Collado (2001) cómo se relacionan los seres, cómo se ven entre sí y la forma en que planean y regulan sus intercambios comunicativos.

En este diálogo directo siempre ha sido necesario que las personas que participan compartan los signos para que exista compresión, es decir que el hecho de que hablen el mismo idioma no significa que exista un igual nivel de entendimiento, además está de por medio un factor básico que es la escucha, no es lo mismo oír que escuchar, elementos que han funcionado a lo largo de los años, pese al aparecimiento de medios de comunicación tecnológicos.

A la comunicación interpersonal se la identifica por el medio social en el que se intercambian los mensajes, por lo que una determinante situacional más importante de este tipo de comunicación es de acuerdo con el número de comunicadores que participan en el intercambio de mensajes, por ejemplo una conversación entre amigos favorece la proximidad física porque les permite el empleo de todos los canales sensoriales, que es el lenguaje no verbal y que hace posible que el mensaje sea directo. (Fernández, 2001).

La comunicación es una forma de relacionarse con las demás personas a través de distintos canales, lenguajes y códigos, según el texto la "Comunicación Humana", (Fernández, 2001) la comunicación es el proceso social primario y por consiguiente crea el mundo en el que ocurre los acontecimientos, es decir, la comunicación es una práctica social que se aprende en diferentes ámbitos, como la familia, escuela, trabajo, en donde se desarrolla principalmente el nivel 
primario de la comunicación que es la interpersonal.

Parte de esta comunicación directa está el lenguaje no verbal que es todo aquello que desborda o trasciende del lenguaje oral o escrito, Flora Davis dice que la comunicación no verbal es básica:

"Las palabras son tan solo el comienzo, pues detrás de las palabras está el cimiento sobre el cual se construyen las relaciones humanas -la comunicación no verbal-, las palabras son hermosas, fascinantes e importantes pero lo hemos sobreestimado en exceso, ya que no representan la totalidad, ni siquiera la mitad del mensaje. Más aún como lo sugirió cierto científico: las palabras pueden muy bien ser lo que emplea el hombre, cuando todo lo demás ha fracasado" (Davis, 1989).

Las palabras o la ausencia de ellas (Magnani, 2008) van acompañadas de un gran número de señales que están relacionadas con la comunicación no verbal, es decir cualquier palabra pronunciada tiene una entonación y va apoyada de una gestualidad, por regla general cuando se está mintiendo o forzando una situación, el cuerpo delata, esto significa que las actitudes no verbales pueden dar pistas para percibir al interlocutor en muchas conversaciones o reuniones.

Los comunicadores se perciben mutuamente como miembros de grupos sociales o culturales particulares, y se atribuyen características determinadas, las cuales se perciben como afines y comunes a todos los miembros de esos grupos (Fernández, 2001:24). Cuando las personas inician un proceso de comunicación, elaboran predicciones acerca de los resultados o consecuencias de las diferentes estrategias de mensajes o de selección alternativas de los mismos.

En la mayoría de los intercambios rutinarios, la elaboración de predicciones se realiza a un bajo nivel de conciencia y es igual a lo que se conoce como una percepción. Sobre lo mencionado, Carlos Fernández cita el saludo como ejemplo, que ya es algo que se lo dice de memoria, ¿cómo estás?, i bien gracias y tú!, pero si el destinatario responde ique te importaj, o no contesta, eso ya genera un malestar en la comunicación cara a cara.

Hay varios factores que contribuyen al establecimiento y mantenimiento de las relaciones interpersonales: la proximidad física, la cual es básica en la relación, las actitudes similares, que influyen de manera intensa en la formación de las relaciones y la complementariedad de necesidades.

Cabe señalar que el sistema de comunicación verbal es el único con ca- 
pacidad para traducir o interpretar otros sistemas, por ejemplo un buen narrador es capaz de crear ambientes, situaciones, atmósferas especiales y temporales mediante la palabra. Al respecto Sebastián
Serrano dice que "no es una hipótesis excesivamente arriesgada afirmar que el lenguaje verbal es el interpretante por antonomasia". (Serrano, 1988, pág. 65).

\section{LA COMUNICACIÓN Y LAS TECNOLOGÍAS}

La comunicación evoluciona día a día a lo largo de la historia; el ser humano ha desarrollado sus formas de relacionarse: desde rudimentarios métodos como la escritura jeroglífica, pasando por la invención de la imprenta y luego con la aparición del teléfono, el cine, la radio, la televisión y la Internet, esta innovación ha sido posible gracias a la tecnología que ha permitido el paso de la sociedad industrial a la actual sociedad de la información, derivada del desarrollo y la combinación de dos tipos de tecnologías que nacieron y despegaron a mediados del siglo XX: las telecomunicaciones y la tecnología informática.

Es menester hacer un poco de historia, sobre la aparición de las nuevas tecnologías, ya en la década del 80 se produjo la expansión masiva del uso del ordenador, que llenó las oficinas para luego extenderse al ámbito doméstico, y evolucionar para instalarse en la sociedad del nuevo milenio llamada como "sociedad de la información y la comunicación", que consiste en un conjunto de cambios en lo económico y social que cambiarán su base material (Castells M., 1995), caracterizada por la introducción generalizada de las nuevas tecnologías de la información y la comunicación en todos los ámbitos de nuestras vidas.

La red de redes se involucró en las vidas de las personas desde los años 90, cuando la computadora se convirtió en un medio de comunicación, que mediante una conexión en la cual se podía contar con internet, permitiendo de esta manera conectarse y conocer culturas, novedades e información a nivel global. A inicios, esa comunicación era limitada, estática y no permitía una interacción, únicamente proporcionaba información a los navegantes de la red.

Más tarde, alrededor del año 2004 aparece la Web 2.0, este nuevo sistema termina con la unidireccionalidad y da lugar a que las personas puedan intercambiar información, de esta forma surge un nuevo modelo de participación y de acción, donde se crean superestructuras informativas en donde 
estos usuarios son los que desarrollan las innovaciones.

Sin embargo, hay quienes creen que el mundo digital limita a las personas en su comunicación interpersonal, se les dificulta relacionarse con los otros, incluso algunos comunicadores han señalado que los nativos digitales tienen en su cabeza un número de caracteres que no les permite hablar más de diez frases cuando están frente a otras personas, el comunicador Ramiro Diez (Diez, 2012), señala que a causa de las nuevas herramientas tecnológicas los jóvenes están desmejorando su lenguaje, presentan dificultades para expresarse en público o para mantener una larga conversación.

En el texto más allá de la Modernidad, dice que la sociedad de la información es la que está distorsionando muchos aspectos, cita como ejemplo las redes familiares que se ven desmejoradas por su destructivo impacto sobre el trabajo de los hombres y mujeres impidiéndoles ejercer sus responsabilidades paternas y maternas. (Gil, 2002)

Según Gil Calvo (2002) con la presencia la Internet y el teléfono celular en el mundo se están presentando nuevas microsituaciones virtuales, entendiéndose como los nuevos contextos de interacción reversible que las redes de interconexión digital están haciendo posible, lo que significa que en esta sociedad de la información están creciendo nuevas máscaras de participación virtual que se ven en los seudónimos que adoptan los cibernautas para navegar. Aparece una generalizada representación ficticia de una prefabricada naturalidad espontánea, que se aprende y se contagia por doquier gracias a la premeditada contemplación de panópticos programas audiovisuales como el "big brother" o los emoticones que intercambian los jóvenes en sus charlas.

Villanueva (Villanueva, 2005), asegura que en el espacio virtual es muy fácil por ejemplo crear una identidad falsa, dice que la sinceridad en la expresión no tiene que ir acompañada por sinceridad en la identificación, añade que al usar la palabra escrita, sin revelar rostros y lugares es fácil ser otra persona, sin mucho esfuerzo.

Estas tecnologías según Adell (Adell, 1997) están cambiando todo y menciona:

\footnotetext{
"Tal vez uno de los fenómenos más espectaculares asociados a este conjunto de transformaciones sea la introducción generalizada de las nuevas tecnologías de la información y la comunicación en todos los ámbitos de nuestras vidas. Están cambiando nuestra manera de hacer las cosas: de trabajar, de divertirnos, de relacionarnos y de aprender. De modo sutil también está cambiando nuestra forma de pensar".
} 
Esto ratifica que las nuevas tecnologías de la información y de la comunicación han llegado y están aquí para quedarse, han modificado de forma sustancial la vida cotidiana de las personas especialmente de aquellas que viven en la ciudad, desde la forma de trabajar, la forma de relacionarse con otras personas o de ocupar el tiempo libre.

En la actualidad la revolución tecnológica trastocó y modificó las viejas estructuras de los medios tradicionalmente llamados de comunicación de masas, la llegada de la radio, la televisión, la utilización de satélites, de la informática, de las complejas tecnologías que en la actualidad configuran cualquier medio por pequeño que sea, ha producido sin duda una nueva manera de enfocar la noticia, de informar en suma de comunicar (Drago, 1990). Surge así una nueva filosofía de interrelacionarse entre individuos, entre emisor y perceptor.

Para Marshall McLuhan los medios electrónicos de información habrían terminado con la intimidad y determinan toda la comprensión del entorno (McLuhan, 1980). Castells en su libro la Galaxia internet, señala que la internet nació en la insólita encrucijada entre la gran ciencia y la cultura libertaria, es decir se desarrolló en un entorno seguro, gracias a los fondos públicos y a un proyecto de investigación orientado hacia el cumplimiento de una misión de interés nacional. (Castells M., 2001)

Los usos de la internet son instrumentales fundamentalmente y están relacionados con las personas a través de sus trabajos, la familia, y la vida cotidiana. Según Castells (2001) el correo electrónico representa más del $85 \%$ del uso de la internet. La actividad social en toda su diversidad se ha apropiado de esta herramienta.

Los que se han involucrado de manera directa son las personas que nacieron con las tecnologías a quienes se les conoce como "nativos digitales" que según Marc Prensky (Prensky, 2009) son los jóvenes que nacieron y crecieron con la red, se caracterizan porque reciben la información de manera muy rápida, son multitareas, eligen textos dinámicos combinados con audio y video, utilizan los hipertextos y son expertos en trabajar en red.

Uno de los elementos principales para la comunicación de los nativos digitales es el chat, que según Joan Mayans, el chat cuenta con elementos que facilitan la comunicación, sin embargo, añade que por ejemplo los emoticones o símbolos que representan los sentimientos y emociones se pueden tomar como una faceta teatral extraordinaria, pero no se iguala a lo que expresa la comunicación cara a cara. (Mayans, 2002) 
Existe el otro grupo de personas que no manejan bien las redes conocidos como inmigrantes digitales, quienes tienen estructuras mentales moldeadas por los procesos paso a paso, prefieren leer en físico el texto, confirmar el mensaje con una persona mediante una llamada y leer el manual de uso de algún equipo antes de usarlo, estas personas son las que tienen edades comprendidas entre los 35 y 55 años que han tenido que adaptarse a una sociedad cada vez más tecnologizada.

Las nuevas tecnologías han planteado parámetros de discriminación a causa de la brecha existente entre los grupos de mayor acceso, en este caso los nativos digitales y los otros que no tienen el conocimiento ni la habilidad para el desempeño en el manejo de las nuevas tecnologías, además hay que resaltar que el desarrollo tecnológico comunicacional presenta una desigualdad entre los países del tercer mundo y el primer mundo, este último ha dominado la industria de la comunicación, información y entretenimiento.(Silva, 2001).

Villanueva (2006), señala que internet usan varios grupos para conversaciones a veces triviales entre desconocidos hasta diálogos de largo aliento entre personas conocidas o desconocidas pero que comparten intereses. Se afirma a menudo que el anonimato es la característica causal que hace de los entornos cibersociales un lugar propicio para la desinhibición y la socialización. Según Reid estos espacios son los adecuados para hablar sin tapujos, que no se atreverían a hacerlo cara a cara (Reid, 1991).Sí es importante señalar que hay una serie de aspectos no verbales que no se pueden transmitir en los "chats".

\begin{abstract}
"Es un nuevo género hiperfilosófico tanto en la forma como en el contenido, todo evento humano mediado directa o indirectamente por las nuevas tecnologías de la información pertenece plenamente a la cibercultura, e influye buena parte de las humanidades y todas las ciencias". (Gutiérrez, 2005)
\end{abstract}

"Se informatizan o se mueren" es una especie de consigna que se asume en los países pobres, necesitado de expandir el consumo informático. (Barbero J., 1987). Según lo que menciona Castells tanto en Estados Unidos como en el mundo en general existe un retraso considerable entre la inversión del hardware tecnológico y la conectividad on line, incluso según varios estudios por ejemplo en la educación existe un serio analfabetismo tecnológico por parte de los profesores, muchos de ellos no reciben capacitación en el manejo de las nuevas tecnologías, esto hace que se dé una gran brecha entre los jóvenes y los adultos (Castells, 2001). 
El uso de la internet tanto en las escuelas como en el ámbito profesional genera desigualdades, desde la educación, el género y el origen étnico. Además las condiciones en las que se está difundiendo la internet en la mayoría de países está provocando una profunda divisoria digital. Los centros urbanos más importantes, las actividades globalizadas y los grupos sociales de mayor nivel educativo están entrando en las redes globales basadas en la internet, mientras que la mayor parte de las regiones y de las personas siguen desconectadas (Castells,2001:290).

\section{CIBERCULTURA}

El mundo está inmerso en la cibercultura que es un concepto que produce cambios epistemológicos y socioculturales, para Gutiérrez (2005), se trata de una forma de hablar, de asimilar, se convierte en una práctica de los seres humanos.

Para Mark Dery, un crítico cultural norteamericano (Dery, 1998) , la cibercultura se posiciona como un movimiento contracultural que ve la convergencia del hombre y la máquina, dice que es la sustitución de la experiencia sensorial por la simulación digital. Entonces la cibercultura significa que el hombre se fundirá con la máquina en un sustancia digital que poco a poco se volverá real dentro del ciberespacio. Cabe resaltar que este término fue acuñado por William Gibson en su novela de ciencia ficción Neuromante, es un nombre que se utiliza para referirse al espacio de las redes (Rheingold, 1996).
Lo que significa que las prácticas sociales de las personas y de los colectivos han cambiado, como lo señala Rocío Rueda Ortiz en su artículo: Cibercultura: metáforas, prácticas sociales y colectivos en red:

"Frente a esta relación entre movimientos sociales y TIC, Valderrama (2008) propone dos aspectos nodales: el papel mediador de estas últimas en los procesos comunicativos y las profundas transformaciones de las dinámicas políticas de la sociedad (que pasan por la crisis estructural de legitimidad y de las prácticas del ejercicio político tradicional). Así, son varias las características de este cambio:

a. Se matiza la centralidad del espacio público urbano de interacción cara a cara, así como la llamada esfera pública, y se promueve una nueva 
provista por la inmaterialidad de las redes electrónicas (ejemplo de ello son espacios como Facebook, Flickr, Myspace, etc.).

b. Las prácticas sociales se constituyen en torno a valores culturales, modos de vida y construcciones de sentido (más allá de intereses de clase o sectoriales) y en oposición a modos de organización y comunicación verticales, burocráticos y rígidos, de ahí que se privilegie la adopción de un tejido organizacional y comunicativo en red.

c. La presencia creciente de colectivos y movimientos sociales de carác- ter global en la Red que no obedecen directamente a regulaciones estatales. (Rueda, 2009).

Así mismo Rueda Ortiz (2009), señala que actualmente se vive en una sociedad con intereses diversos y heterogéneos, cuyas generaciones están envueltas en un mundo visual y digital dejando de lado las interacciones con la familia. Esto lo justifica Barbero (Barbero M., 2005) al señalar que estas simbiosis se produce a causa de que las tecnologías reconfiguraron los espacios urbanos y las formas de habitar de las personas.

\section{ADICCIÓN A LAS TECNOLOGÍAS}

Los nativos digitales tienen nuevas formas de interactuar, percibir y conocer a través de las tecnologías que les posibilita abrir su mundo de otra manera que no es la comunicación cara a cara. Rueda Ortiz afirma que en este mundo tecnológico se están generando disturbios, angustias, sufrimientos y patologías que lo viven los jóvenes.

A nivel clínico, ciertas conductas como el abuso de las nuevas tecnologías, el juego patológico, la hipersexualidad, el abuso de la Internet etc, pueden considerarse como adicciones, los síntomas que generan son similares como cuando consumen droga, por ejemplo estar conectado a la red y no poder vivir sin ella puede actuar como un estimulante que produce cambios fisiológicos en el cerebro. En algunas personas estar en la Internet es una droga que no la pueden dejar y que clínicamente genera un humor depresivo, irritabilidad, inquietud psicomotriz, deterioro de la concentración y trastornos del sueño (Echeburúa, 2009).

Según Echeburúa lo que se define como una conducta adictiva es la 
pérdida de control por parte de la persona, una dependencia tal que altera totalmente su vida cotidiana. Lo que caracteriza a este tipo de adicción sin droga es la forma de relación que la persona establece con la actividad que realiza.

Las nuevas tecnologías abrieron un campo de amplísimas posibilidades a todos los niveles, la mayoría positivas. Sin embargo, entrañan también riesgos. El problema no reside en la tecnología en sí, sino, como ocurre con otros ámbitos, en el uso que hacen las personas de ella. La Organización Mundial de la Salud (OMS) (García, 2014) señala que una de cada cuatro personas sufre trastornos de conducta relacionados con las nuevas adicciones. Se puede hablar de adicción en todos aquellos casos en los que la situación interfiere en la vida diaria, o cuando se practica esa afición para no pasarlo mal.

Entre las señales de alerta que pueden indicar una adicción se destacan las siguientes:
- Pérdida de tiempo de estudio, con o sin malos resultados académicos.

- Deterioro o menor dedicación a las relaciones interpersonales y a otras actividades de ocio.

- Incremento de las conductas que implican agresividad.

- Sufrir ansiedad, irritabilidad, incluso malestar físico en caso de no poder utilizar el objeto de la adicción. (OMS)

Las nuevas tecnologías han pasado a formar parte de las denominadas adicciones psicológicas o adicciones sin drogas. Este comportamiento de adicción impide que los jóvenes desarrollen sus habilidades sociales, hace que sean hipersensibles a los juicios y acrecienta sus sentimientos de inseguridad. En estos casos la familia debe prestar atención a los primeros signos de alarma que se asocian al comportamiento adictivo, como son la tendencia al aislamiento, la ruptura de las relaciones sociales, el fracaso escolar o la agresividad.

\section{MÉTODO}

La presente investigación quiere exponer un problema latente y encontrar respuestas a una pregunta específica:

¿Cuáles son las dificultades que se generan en la comunicación interpersonal entre los jóvenes universitarios y personas de otras generaciones a causa del uso de las TICS?

El método que se usó fue el hipotético-deductivo, se planteó una hipótesis a partir de un problema que se evidencia en la comunicación directa, la 
justificación de este método está dado a partir del surgimiento de un problema. Estos intentos de respuestas son conjeturas, suposiciones que en ciencia se denominan hipótesis.

La hipótesis planteada fue:

La gran mayoría de actividades que realizan diariamente los jóvenes universitarios (nativos digitales) lo hacen con el uso TICS, generando dificultades en la comunicación cara a cara con personas de otras generaciones.

A la hipótesis se definieron las siguientes variables las cuales se operacionalizaron desde la definición, dimensión de las variables y se determinaron los indicadores que permitieron elaborar la encuesta que es una de las técnicas que se utilizó.

Las variables dependientes fueron:

1. Nativos digitales de la PUCE, son personas nacidas a partir de 1980, son usuarios permanentes de las "tics", con una habilidad consumada, los indicadores de esta variable fueron edad, carrera y el semestre que cursa;

2. Uso extensivo de las "TICS" de los nativos digitales de la PUCE para comunicarse y realizar sus actividades académicas y no académicas, cuyas dimensiones de las variables fueron las actividades académicas como estudiar solos o en grupo, clases, prácticas, trabajos en grupo, biblioteca y las no académicas que son la familia, amigos, pares, otras personas. Los indicadores que se usaron fueron:

- Tiempo que dedica a estudiar con las "tics"

- Tiempo que dedica a estudiar en grupo con las "tics"

- Usa en clases las "tics"

- Tiempo que dedica a las prácticas con las "tics"

- Tiempo que dedica para hacer trabajo en grupo con las "tics",

- Tiempo de uso de la biblioteca virtual

- Tiempo que dedica a con la familia

- Tiempo que dedica con pares

- Tiempo que dedica con otras personas

3. Comunicación cara a cara que es la interacción entre dos o más actores sociales que se da en un espacio y tiempo determinado, donde intervienen los lenguajes verbal y no verbal en un porcentaje de 35 y $65 \%$ respectivamente. Las dimensiones de esta variable fueron las actividades académicas como estudiar solos, en grupo, clases, prácticas, trabajos en grupo, biblioteca y las o académicas como la familia, amigos, pares, otras personas cuyos indicadores fueron:

- Tiempo que dedica a conversar cara a cara con compañeros, pares, docentes, administrativos 
- Tiempo que dedica a conversar con familiares, amigos, otras personas
La variable independiente fue:

- La existencia de nativos digitales que incide o afecta a las otras variables

\section{METODOLOGÍA}

Esta investigación nos ayuda a esclarecer los cambios que está generando en la comunicación interpersonal cara a cara entre los jóvenes y personas de otras edades, el objetivo es identificar las diferencias unos de otros con la correspondiente delimitación de su alcance actual. En la investigación es imprescindible establecer una delimitación del alcance conceptual y aspectos definidores que permitan acotar el terreno y apreciar, además su enorme complejidad en los cambios que está produciendose.

La observación y análisis se amplía a los nuevos ámbitos comunicativos, que se generan en los procesos interactivos para ello se ha trabajado con la población estudiantil de la PUCE de pregrado a través del uso de la técnica de la encuesta, la propuesta es cualitativa en cuanto se pretende detectar el nuevo fenómeno de comunicación frente a lo que ha sido la comunicación interpersonal cara a cara.

La población estudiantil de la PUCE es extensa, hasta agosto del 2012 la PUCE contó con 10.170 estudiantes distribuidos de la siguiente forma:
- Pregrado:

9.077

- Postgrado:

816

- No definido (cursos

abiertos y estudiantes

de convenio):

277

Los 9077 estudiantes de pregrado de la PUCE fueron el universo de estudio, entendiéndose por universo como la totalidad de elementos bajo consideración, que permitirán arrojar las conclusiones (Méndez, 1998). Se calculó el porcentaje de estudiantes que representa cada facultad en relación con el total de estudiantes de la Universidad. (Anexo 1)

De los 9.077 estudiantes de la PUCE, las facultades que más representación mantienen son la de Ciencias Administrativas y Contables con el 16.5\%, la de Medicina con el 11.62\%, la de Ciencias Humanas con el 10.47\%, la de Arquitectura, Diseño y Artes con el $10.16 \%$ y la de Ingeniería con el 9.01\%.

Con el fin de que la muestra sea representativa y mantenga un nivel adecuado de confiabilidad y determinar un margen de error aceptable se definió como muestra representativa un 10\% del 
universo total, más un 0.3\% como margen de error, lo que significa un número de encuestas totales de 955 unidades.

Se aplicó el porcentaje obtenido por facultad a la muestra determinada. Para inferir a partir de esa muestra los resultados del cuestionario de la encuesta, de tal manera que las encuestas se aplicaron por facultad. (Anexo 2)

Se realizaron las encuestas a los estudiantes de las distintas facultades, durante un mes, con la ayuda de dos estudiantes de comunicación que recogieron directamente las 955 encuestas de alumnos de las distintas unidades académicas de la PUCE.

Las técnicas utilizadas fueron:

a) Investigación bibliográfica con el objetivo de determinar fuentes confiables para el análisis de contenido tanto cualitativo como cuantitativo,

b) Entrevistas a expertos que han estado involucrados en investigaciones similares o viven conflictos en la comunicación con los jóvenes universitarios. Los entrevistados fueron:
- Ramiro Diez comunicador

- María del Carmen Cevallos comunicadora/docente

- Wladimir Sierra Sociólogo/Docente

c) Encuesta: la encuesta se elaboró de acuerdo con los indicadores que se determinaron en las variables, la misma fue sometida a una prueba piloto, y se realizó una encuesta previa a 10 estudiantes de la PUCE considerados nativos digitales: 3 de comunicación, 2 de derecho, 3 de medicina, 2 de arquitectura, convirtiéndose en una fase esencial para la aplicación de la encuesta final, esta práctica previa aportó para detectar si existía alguna dificultad o ambigüedad en cuanto a las preguntas, estructura o finalidad.

El grupo de la encuesta piloto proporcionó no solo una estimación del tiempo necesario para responder las preguntas, sino que comentaron las dificultades que encontraron en la encuesta, sugerencias que fueron acogidas considerando que los encuestados fueron parte del universo de estudio.

\section{RESULTADOS}

La muestra de 955 estudiantes, estuvo distribuida en alumnos nacidos entre los años 1986 y 1995, es decir edades comprendidas entre 17 y 26 años. El 
$75 \%$ de los alumnos encuestados tenían entre 19 y 22 años de edad; pertenecieron al género femenino en el $48.8 \%$, al género masculino en el $50.05 \%$, en tanto que respondieron "otro" 11 alumnos que representan el $1.15 \%$ de la muestra.

Es importante enfatizar que el $65 \%$ del total pertenecieron a los primeros cinco niveles de las carreras que cursan, mientras el $35 \%$ restantes eran estudiantes de sexto a décimo nivel. Para el objeto de este estudio, estos datos dieron mayor fiabilidad a los resultados.

Los estudiantes en forma individual dedican varias horas para estudiar a través de las "tics", un $63 \%$ dedican de una a dos horas a estudiar con el uso de las "TICs", mientras que el $32 \%$ utiliza más de tres horas. Solamente el $3.35 \%$ contesta que no utiliza las "TICS" para estudiar en forma individual.

El estudio en grupo con el uso de las TICs, es utilizado por los estudiantes en gran proporción, hasta una hora la utilizan el $28 \%$ de estudiantes, hasta dos horas el $32 \%$, y más de tres horas el $22 \%$ de los mismos. No estudian en grupo con el uso de las tecnologías de la información un número de 160 estudiantes, que representa el $16.75 \%$ de la muestra.

Al preguntar el número de horas que los estudiantes reciben clases con el uso de las tecnologías de información, el $22 \%$ de los mismos respondieron que no utilizan estas herramientas. El $78 \%$ restante sí reciben sus clases con la utilización de estos medios.

En la pregunta de cuántas horas dedica al día para hacer sus pasantías con el uso de las "tics", más de la mitad de los estudiantes encuestados respondieron que no las utilizan. Del $50 \%$ restante, el $13 \%$ utilizan hasta una hora, el $19 \%$ hasta dos horas y el $16 \%$ más de 3 horas.

En relación con la pregunta que tiene que ver con el promedio de horas que duermen en la noche los jóvenes encuestados, se observa que un 36,44\% duerme alrededor de seis horas, y un $27 \%$ duerme alrededor de siete horas, observándose que están dentro del rango recomendado por los médicos para tener un buen descanso y poder rendir sin problemas al día siguiente.

En la pregunta relacionada con el número de horas que dedican los jóvenes nativos digitales a conversar con familiares por Internet se observa que un buen porcentaje de jóvenes no conversan por Internet con sus familiares, esto equivalió en la encuesta al 51,20 \%, mientras que el $23.87 \%$ conversa cerca de una hora por Internet con su familia, esto ratifica la hipótesis planteada al señalar que estos jóvenes están con las nuevas tecnologías para satisfacer sus intereses antes que conversar con personas cercanas como es la familia. 


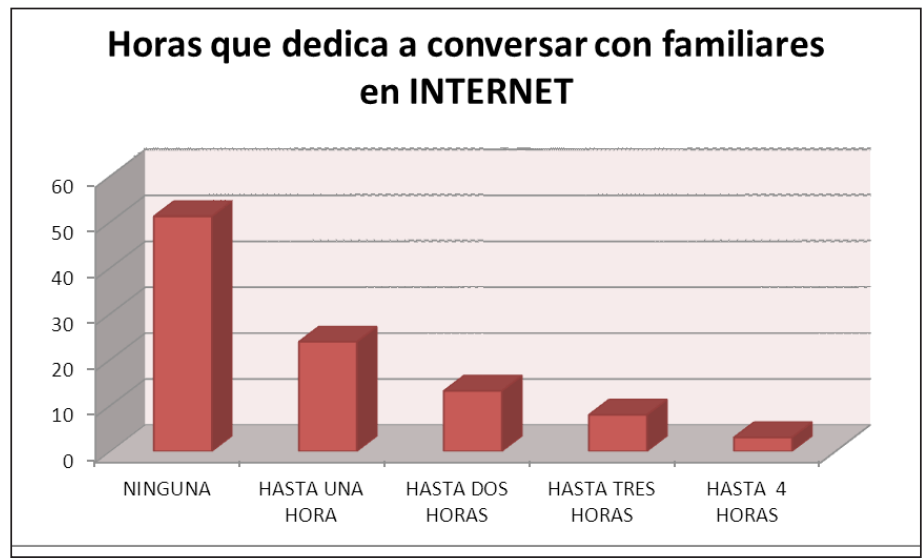

Elaboración propia

La pregunta relacionada con el número de horas que dedican al día para conversar con amigos mediante Internet la encuesta dice que el 29,53\% dedica una hora para conversar con sus amigos, el $28.06 \%$ dedican dos horas, el 16,65\% ninguna hora, y el $12.67 \%$ conversa cuatro horas con sus pares, demostrando que el uso de Internet, de las nuevas tecnologías si lo usan para relacionarse, pero también tiene otros fines como buscar información, entretenerse o jugar para ocupar su tiempo libre.

La pregunta relacionada con el número de horas que dedican al día para conversar con otras personas mediante la Internet revela que el $50.68 \%$ no dedican ninguna hora para relacionarse con personas que no conocen, en cambio el $18.22 \%$ dedica una hora para hacer este tipo de conversaciones, y el $17.80 \%$ lo hace dos horas al día.

A diferencia de la pregunta anterior, los jóvenes sí dedican tiempo para conversar cara a cara con sus pares o amigos, por ejemplo el 31.83\% dedica dos horas al día para conversar con sus amigos, el 23.14\% tres horas, el 21.47 una hora, demostrando que sí mantienen una conversación cara a cara pero con sus pares antes que con personas de otras edades, o mayores que ellos.

En la pregunta relacionada con la interacción con los docentes, la mayoría dijo que es escasa de acuerdo con los resultados, el $52.46 \%$ de los encuestados dijeron que no conversan a ninguna hora cara a cara con los profesores, apenas el $27.54 \%$ lo hace una hora al día, y el $13.93 \%$ dos horas, lo que demuestra que 
a los jóvenes muy poco les interesa interactuar con los docentes porque al ser nativos digitales la información adicional que requieren de algunas materias lo obtienen a través de la red.

Un buen porcentaje de jóvenes encuestados equivalente al $46.70 \%$ no conversan cara a cara a ninguna hora con personas de otras edades, el $26.39 \%$ lo hace una hora al día, y el $18.01 \%$ dedica dos horas, lo que evidencia que los jóvenes universitarios son como se ha dicho a lo largo de la investigación que son nativos digitales que su vida, relaciones, cultura, cotidianidad gira alrededor de las nuevas tecnologías.

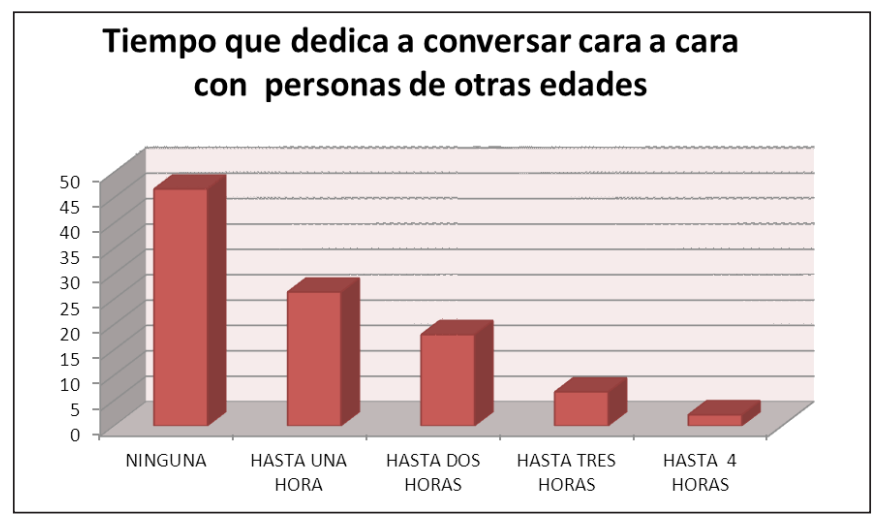

Elaboración propia

El $32.25 \%$ dedica una hora al día para conversar con sus familiares, el $28.27 \%$ dos horas, el $24.29 \%$ ninguna hora lo que se deduce que es muy poco el tiempo que los jóvenes universitarios destinan un tiempo de sus actividades diarias para conversar con las personas más cercanas, ratificando que los jóvenes tienen como prioridad las nuevas tecnologías y conocer otros horizontes mediante la red, abandonando casi de manera completa el contacto con la gente más cercana a ellos. 


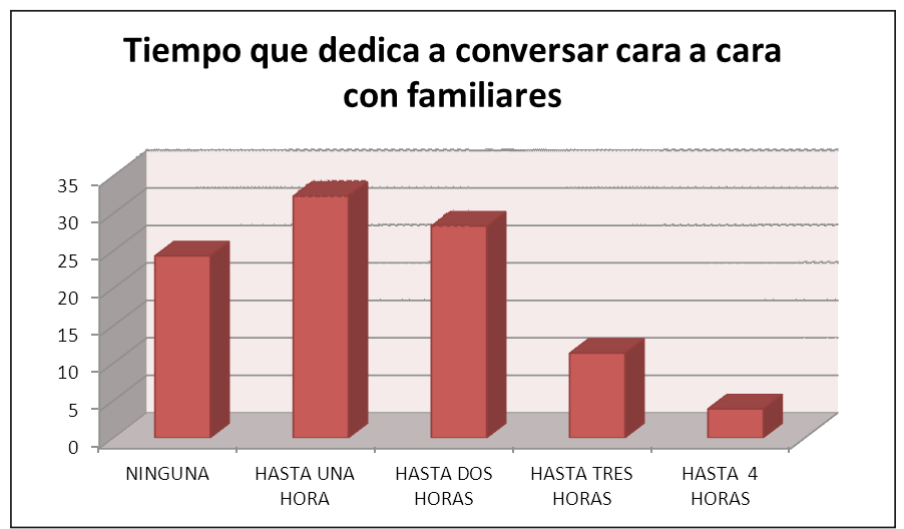

Elaboración propia

\section{CONSIDERACIONES FINALES}

Hoy en día los jóvenes a nivel mundial ven a la red como una forma más rápida de comunicarse con sus pares y personas de otros lugares, para lo cual cuentan con variados equipos tecnológicos como la computadora con su respectiva red de Internet, el ipood, ipad, el celular, las tabs, los dispositivos móviles, etc, equipos que se han convertido en su medio principal de expresión generando una dependencia casi total de la tecnología.

De acuerdo con esta investigación se confirma que ha cambiado el entorno social de los jóvenes de una manera un tanto distorsionada, volviendo a lo virtual como elemento esencial de su vida y desplazando la convivencia interpersonal hacia un segundo plano.
En algunos países -tales como el Reino Unido, los Estados Unidos y partes del Asia oriental- existen datos cuantitativos y cualitativos sobre las formas en que los jóvenes usan las nuevas tecnologías, y estos datos han comenzado a revelar cómo los medios electrónicos están cambiando las costumbres de la juventud. (John Palfrey, 2011)

Si bien es cierto que el surgimiento de las nuevas tecnologías configura un nuevo espacio social donde las personas especialmente los jóvenes tienen la posibilidad de acceder a contenidos personalizados, información mundial, enlaces, páginas interactivas, redes, 
conversaciones, y aplicaciones para que participen en la Web, por ejemplo en fenómenos multifacéticos, como es el caso de los "blogs", también es cierto que la comunicación cara a cara según varios estudiosos permite que los seres humanos manejemos la comunicación verbal y no verbal, convirtiéndose esta última en un código que lo entendemos todos y lo comprendemos por su aporte simbólico.

Varios estudios sobre comunicación realizados en los Estados Unidos (Birdwhistell), aseguran que del $100 \%$ de la comunicación cara a cara, el 35 $\%$ es comunicación verbal y el 65 \% no verbal; el verbal se usa para comunicar información y el no verbal para comunicar estados y actitudes personales. (Knapp, 2012)

La comunicación es una práctica colectiva expresada a través de los diversos lenguajes que muestran sentimientos, emociones, pensamientos, experiencias, formas simbólicas y de significación que se reflejan especialmente a través de los lenguajes verbal y no verbal. Este tipo de comunicación interpersonal, hoy en día, es reemplazada por otros lenguajes especialmente el virtual, que genera nuevas prácticas cotidianas de comunicación especialmente en los jóvenes considerados nativos digitales, por ejemplo, el chat y los mensajes de texto han provocado dificultades de comunicación interpersonal entre los jóvenes nativos digitales y los adultos en general.

De acuerdo con lo investigado se concluye que los jóvenes universitarios no se desamparan a lo largo del día de su teléfono celular, tablets, y demás aparatos tecnológicos. Mediante conversaciones mantenidas con los estudiantes, mencionan que el celular se ha convertido en una parte principal de su vida, su cotidianidad se mueve alrededor de las tecnologías. En el texto más allá de la Modernidad, su autor Gill Calvo, menciona que en esta nueva era, las tecnologías traen muchas ventajas pero también generan distorsión en muchos aspectos, y cita como ejemplo las redes familiares que se ven desmejoradas por su destructivo impacto.

Como se mencionó al inicio del artículo la comunicación no verbal actúa en un porcentaje alto dentro de la comunicación cara a cara, lo que significa que este tipo de lenguaje es fundamental para percibir al interlocutor desde sus sentimientos, sensaciones, emociones, poder observar los gestos, la mirada, la expresión corporal y la espontaneidad de la persona para expresarse, por ejemplo cualquier palabra pronunciada tiene una entonación y va apoyada de una gestualidad.

Muchas veces se puede detectar que la persona está mintiendo o forzan- 
do una situación, el cuerpo nos delata, esto significa que las actitudes no verbales pueden dar pistas para percibir al interlocutor en muchas conversaciones o reuniones, al existir la mediación a través de los instrumentos tecnológicos en las conversaciones se desconoce si lo que dicen es cierto o falso porque los símbolos conocidos como "emoticons" que se usan no remplazan en ningún caso la conversación directa.

Las tecnologías han generado espacios de nuevas relaciones sociales entre los jóvenes, a nivel mundial o de orden planetario, estos cambios se observan en los comportamientos y la cultura de los jóvenes, lo que provoca poco interés en relacionarse con personas cercanas en los espacios donde se desenvuelven tanto en la familia como en la universidad. Los jóvenes que fueron el universo de estudio prefieren mantener conversaciones con sus pares a través de la red y dejar de lado las relaciones interpersonales.

Como lo señala Adell, citado en este artículo, las tecnologías están cambiando las rutinas y los hábitos de vida diaria, las formas de relacionarse, y de aprender, ante el uso desmesurado de los medios tecnológicos van desapareciendo tradiciones sociales importantes de nuestra cultura que se han caracterizado por el contacto físico, el mirarse a los ojos, el apretón de manos, etc. Al encontrarse inmerso en el mundo de los medios tecnológicos, van adaptando falsas apariencias, incluso sentimientos y emociones, no comparten la interacción familiar y se produce una brecha y un distanciamiento con otras generaciones.

En el caso específico de la Pontificia Universidad Católica del Ecuador el $99.9 \%$ usa las nuevas tecnologías, de acuerdo con los resultados de las encuestas, incluso en consultas hechas a la Dirección de Promoción de la PUCE señalan que los estudiantes desean recibir información de la universidad mediante la web, convirtiéndose en un indicador que confirma lo señalado.

En suma esta investigación invita a la reflexión de las y los ciudadanos tomando en cuenta que la comunicación cara a cara enriquece los vínculos humanos a través del intercambio de emociones, sentimientos, sensaciones y significados, concluyendo que la tecnología no significa necesariamente progreso, ofrece oportunidades pero también genera problemas de comunicación en la cotidianidad de las personas.

Las claves para superar este tipo de dependencias pasa por solucionar los problemas de base, fomentar la comunicación familiar, restablecer la confianza con los padres y los amigos y aceptar la imagen corporal, que es uno de los factores que más contribuyen a la adicción a las tecnologías. 


\section{ANEXOS}

Anexo 1 Porcentaje a considerar para definir la muestra

\begin{tabular}{|r|r|r|r|}
\hline \multicolumn{2}{|c|}{ DETALLE } & \multicolumn{2}{c|}{ TOTAL ESTUDIANTES } \\
\cline { 2 - 4 } & \multicolumn{1}{|c|}{ No. } & $\%$ \\
\hline 1 & Escuela de Bioanálisis & 222 & $2 \%$ \\
\hline 2 & Escuela de Trabajo Social & 76 & $1 \%$ \\
\hline 3 & Facultad de Arquitectura, Diseño y Artes & 924 & $10 \%$ \\
\hline 4 & Facultad de Ciencias Administrativas y Contables & 1,500 & $17 \%$ \\
\hline 5 & Facultad de Ciencias de la Educación & 239 & $3 \%$ \\
\hline 6 & Facultad de Ciencias Exactas y Naturales & 276 & $3 \%$ \\
\hline 7 & Facultad de Ciencias Filosóficas y teológicas & 116 & $1 \%$ \\
\hline 8 & Facultad de Ciencias Humanas & 953 & $10 \%$ \\
\hline 9 & Facultad de Comunicación Literatura y Lingüística & 630 & $7 \%$ \\
\hline 10 & Facultad de Economía & 504 & $6 \%$ \\
\hline 11 & Facultad de Enfermería & 520 & $6 \%$ \\
\hline 12 & Facultad de Ingeniería & 822 & $9 \%$ \\
\hline 13 & Facultad de Jurisprudencia & 510 & $6 \%$ \\
\hline 14 & Facultad de Medicina & 1,054 & $12 \%$ \\
\hline 15 & Facultad de Psicología & 731 & $8 \%$ \\
\hline & TOTAL & 9,077 & $100 \%$ \\
\hline
\end{tabular}


Anexo 2: porcentaje de la muestra

\begin{tabular}{|r|l|r|r|}
\hline \multicolumn{2}{|c|}{ DETALLE } & \multicolumn{1}{c|}{ TOTAL } & \multicolumn{1}{c|}{$\begin{array}{r}\text { OOTAL } \\
\text { MUESTRA }\end{array}$} \\
\cline { 2 - 4 } 1 & Escuela de Bioanálisis & $2 \%$ & 23 \\
\hline 2 & Escuela de Trabajo Social & $1 \%$ & 9 \\
\cline { 1 - 3 } 3 & Facultad de Arquitectura, Diseño y Artes & $10 \%$ & 97 \\
\hline 4 & Facultad de Ciencias Administrativas y Contables & $17 \%$ & 158 \\
\hline 5 & Facultad de Ciencias de la Educación & $3 \%$ & 25 \\
\hline 6 & Facultad de Ciencias Exactas y Naturales & $3 \%$ & 29 \\
\hline 7 & Facultad de Ciencias Filosóficas y teológicas & $1 \%$ & 12 \\
\hline 8 & Facultad de Ciencias Humanas & $10 \%$ & 100 \\
\hline 9 & Facultad de Comunicación Literatura y Lingüística & $7 \%$ & 66 \\
\hline 10 & Facultad de Economía & $6 \%$ & 53 \\
\hline 11 & Facultad de Enfermería & $6 \%$ & 55 \\
\hline 12 & Facultad de Ingeniería & $9 \%$ & 86 \\
\hline 13 & Facultad de Jurisprudencia & $6 \%$ & 54 \\
\hline 14 & Facultad de Medicina & $12 \%$ & 111 \\
\hline 15 & Facultad de Psicología & $8 \%$ & 77 \\
\hline & Total & & 955 \\
\hline
\end{tabular}




\section{BIBLIOGRAFÍA}

Adell, J. (noviembre de 1997). EDUTEC, Revista Electrónica de Tecnología Educativa, no 7 . Recuperado el 12 de 06 de 2012, de http://nti.uji. es/docs/nti/Jordi_Adell_EDUTEC. html

Barbero, J. (1987). De los medios a las mediaciones. Barcelona: Gilli Gustavo S.A.

Barbero, M. (2005). Cultura y nuevas mediaciones tecnológicas. Bogotá: Convenio Andrés Bello.

Castells, M. (1995). La ciudad informacional: Tecnologías de la Información, reestructuración económica y el proceso urbano regional,. Madrid: Alianza Editorial.

Castells, M. (2001). La Galaxia Internet. Barcelona: Plaza y Jánes Editores.

Cevallos, M. d. (19 de abril de 2012). Cordiandora de Laboratorio de medios UDLA. (J. Leiva, Entrevistador)

CIESPAL. (2012). Jóvenes y tics. CAHSQUI, $4,5,6,7,8,9,10$.

comunicación., E. I. (1991). conferencia del profesor Marío Bunge. En Innovación tecnológica y comunicación (pág. 25 a 41). Madrid : Siruela. S.A.

Davis, F. (1989). El lenguaje de los gestos . Buenos Aires: AMECE.

Dery, M. (1998). Velocidad de escape. Ma- drid: Siruela.

Diez, R. (25 de abril de 2012). Comunicador . (J. Leiva, Entrevistador)

Drago, T. (1990). Introducción . En Innovación tecnológica y comunicación (pág. 13). Madrid : Siruela S.A.

Echeburúa, E. (2009). Adicciones a las nuevas tecnologías. Madrid: Pirámide.

Echeburúa, E. y. (2009). Adicción a las nuevas tecnologías en adolescentes y jóvenes. Madrid: PIRAMIDE.

Fernández, C. (2001). La comunicación humana en el mundo contemporáneo. Mexico: Mc graw-hill.

García, P. (23 de 05 de 2014). Los adolescentes: uso y abuso de las nuevas tecnologías y redes sociales. Recuperado el 24 de 11 de 2106, de avanza Psicología: http://www. avanza-psicologia.es/adolescentes-uso-abuso-nuevas-tecnologias-redes-sociales/

Garitaonandia, C. (2007). uso de la Internet de los jóvenes:hábitos, riesgos y control parental. Madrid.

Gil, E. (2002). Quiebra y reconstrucción de las narrativas vitales. En ¿Más allá de la Modenidad? (pág. 273). Madrid: CIS.

Guitiérrez, M. (2005). Internet y Libertad. Sevilla: C/S.

Gutiérrez, M. (2005). Internet y Libertad. Sevilla: C/S. 
Hernandez, R. (2003). Metodología de la Investigación. México: Mc Graw Hill.

INEC. (01 de 09 de 2016). Tecnologías de la Información y comunicación (TIC'S) 2015. Recuperado el 20 de 12 de 2016, de Estadísticas sociales TIC 2015: http:// www.ecuadorencifras.gob.ec// documentos/web-inec/Estadisticas_Sociales/TIC/2015/Presentacion_TIC_2015.pdf

John Palfrey, U. G. (17 de 01 de 2011). Los nativos digitales. Recuperado el 27 de 06 de 2015, de UNICEF: https://www.unicef.org/spanish/ sowc2011/pdfs/Los-nativos-digitales.pdf

Knapp, M. L. (11 de 03 de 2012). La comunicación no verbal.El cuerpo y el entorno. Recuperado el 13 de 12 de 2016, de Federación Latinoamericana de Semiótica: http:// www.felsemiotica.org/site/ wp-content/uploads/2014/10/ Knapp-Mark-L.-La-comunicaci\%C3\%B3n-no-verbal.-El-cuerpo-yel-entorno.pdf

Magnani, E. (2008). Historia de la comunicación. Argentina : Capital intelectual.

Mayans, J. (2002). Género Chat. Barcelona España : Gedisa, S.A.

McLuhan, M. (1980). La compresión de los medios como las extensiones del hombre. México: Diana.

Mendez, C. (1998). Metodologías Guía para elaborar diseños de investigación en ciencias económicas, contables y administrativas. Bogotá: Mc.GRAW-HILL.INTERAMERICANA S.A.

Pereira, A. (2002). Semiótica y comunicación. Quito: FEDUCOM.

Prensky, M. (14 de 05 de 2009). nativos-digitales-parte nativos digitales, inmigrantes digitales. Recuperado el 20 de 06 de 2014, de blog de recursos de aprender a pensar: http://recursos.aprenderapensar. net/files/2009/04/nativos-digitales-parte1.pdf

Real Academia Española . (2014). Diccionario de la lengua española. Obtenido de http://dle.rae.es/?id=Ok8i4DT

Reid, E. (1991). Electrópolis. Observatorio para la cibersociedad, 7-15.

Rheingold. (1996). La comunidad virtual, una sociedad sin fronteras. BarceIona: Gedisa.

Rueda, R. (23 de 04 de 2009). http://www. scielo.org.co/Cibercultura: metáforas,prácticas sociales y colectivos en red. Recuperado el 23 de 07 de 2012, de scientific electronic librery on line: http://www.scielo. org.co/pdf/noma/n28/n28a2

Sally Burch, Oswaldo León. (2004). Se cayó el sistema, enredos de la So- 
ciedad de la información. Quito: Unicef. (17 de 01 de 2011). tecnología. Alai. Obtenido de http://www.unicef.

Serrano, S. (1988). La semiótica. Barceloorg/spanish/sowc2011/pdfs/ na: Montesinos Editor S.A. Los-nativos-digitales.pdf

Sierra, W. (19 de abril de 2012). sociólogo. (J. Leiva, Entrevistador)

valerino, E. Y. (2010). Metodlogia de la comunicación. México : Trillas .

Silva, U. (2001). Impacto social de las tecnologías de información y comunicación en el espacio local. Villanueva, E. (2005). Comunicación interpersonal en la era digital. Bogotá: Norma S.A.

En FLACSO, Internet y Sociedad en América latina y el caribe (págs. 279-307). Quito: IDRC. 